\title{
Impact of the COVID-19 outbreak on the profession and psychological wellbeing of radiologists: a nationwide online survey
}

Francesca Coppola ${ }^{1 \dagger}$, Lorenzo Faggioni ${ }^{2^{*}}$ (0), Emanuele Neri ${ }^{2,3}$, Roberto Grassi ${ }^{4}$ and Vittorio Miele ${ }^{5}$

\begin{abstract}
Background: The COVID-19 outbreak has played havoc within healthcare systems, with radiology sharing a substantial burden. Our purpose is to report findings from a survey on the crisis impact among members of the Italian Society of Medical and Interventional Radiology (SIRM).
\end{abstract}

Methods: All members were invited to a 42-question online survey about the impact of the COVID-19 outbreak on personal and family life, professional activity, socioeconomic and psychological condition. Participants were classified based on working in the most severely affected Italian regions ("hot regions") or elsewhere.

Results: A total of 2150 radiologists joined the survey. More than $60 \%$ of respondents estimated a workload reduction greater than $50 \%$, with a higher prevalence among private workers in hot regions $(72.7 \%$ vs $66.5 \%$ elsewhere, $p=0.1010)$. Most respondents were concerned that the COVID-19 outbreak could impact the management of nonCOVID-19 patients and expected a work overload after the crisis. More than 40\% were moderately or severely worried that their professional activity could be damaged, and most residents believed that their training had been affected. More than $50 \%$ of respondents had increased emotional stress at work, including moderate or severe symptoms due to sleep disturbances, feeling like living in slow motion and having negative thoughts, those latter being more likely in single-living respondents from hot regions [log OR 0.7108 (CI95\% $0.3445 \div 1.0770), p=0.0001$ ].

Conclusions: The COVID-19 outbreak has had a sensible impact on the working and personal life of SIRM members, with more specific criticalities in hot regions. Our findings could aid preserving the radiologists' wellbeing after the crisis.

Keywords: COVID-19, Radiology, Workload, Psychological disorders, Online survey

\section{Key points}

- The COVID-19 crisis was estimated to reduce radiologists' workload by up to $>50 \%$.

- The crisis was deemed to impact management of non-COVID-19 patients.

\footnotetext{
*Correspondence: Ifaggioni@sirm.org

${ }^{\dagger}$ Francesca Coppola, Lorenzo Faggioni contributed equally to the manuscript preparation

${ }^{2}$ Department of Translational Research, University of Pisa, Via Roma, 67,

56126 Pisa, Italy

Full list of author information is available at the end of the article
}

- Surveyed residents believed that their training had been affected.

- More than $50 \%$ of respondents experienced psychological symptoms.

\section{Introduction}

In January 2020, a novel coronavirus strain called Severe Acute Respiratory Syndrome Coronavirus 2 was identified as the causative agent of several cases of interstitial pneumonia referred to as Coronavirus 2019 disease (COVID-19), which made its first appearance in Wuhan, China in December 2019 and has spread worldwide since 
then. As known, a rapidly growing number of individuals have been infected worldwide, and this unprecedented situation has put an overwhelming pressure on healthcare systems, resulting into disruption of regular workflow and potentially worse non-COVID-19 outcomes due to late or missing diagnosis and/or treatment [1-3].

Among medical specialties, radiology has shared a substantial burden due to its pivotal role in managing COVID-19. The sudden need to divert substantial resources to face the crisis has generated further issues, including lower imaging volumes, delayed procedures for non-COVID-19 patients, and curtailed educational and research activities [4-7]. Major radiological societies have striven to support radiologists by endorsing specific activities, including the production of guidelines based on available evidence and encouraging multicentric data collection and sharing [8-10].

Our purpose is to report and analyze the results of an online survey aimed to assess the impact of the COVID19 outbreak on the working and personal life of members of the Italian Society of Medical and Interventional Radiology (SIRM).

\section{Methods and materials}

This survey was launched by the Imaging Informatics Chapter of SIRM as a SIRM initiative to assess the impact of the COVID-19 outbreak among its members. The survey was conducted using similar methods to previous SIRM surveys [11-14].

Based upon suggestions from a multidisciplinary panel, two radiologists of the Imaging Informatics Chapter of SIRM (F.C., L.F.) created the survey using the SurveyMonkey platform (www.surveymonkey.com). The survey consisted of 42 questions (of which 36 were single choice and 6 multiple choice), grouped into five sections about demographic information and impact of the COVID-19 crisis on personal and family life, professional activity, socioeconomic condition and psychological status (Additional file 1). Each member received a personal email invitation to join the survey, which could be accessed only once over a period of one week.

Data were analyzed using SurveyMonkey Statistical Tool and a statistical software package (GraphPad Prism v. 5, www.graphpad.com). The association between survey replies expressed as categorical variables in the four regions most severely hit by the COVID-19 outbreak [15] (henceforth named "hot regions") versus other regions was evaluated using the Chi-square test. Differences in the distributions of replies pertaining to emotional and interpersonal issues (i.e. Questions \#9, 10, 20, 22, 23 and from 31 to 42) between male and female respondents in hot versus other regions, and between single-living and other respondents in hot versus other regions, were assessed in terms of log-odds ratios and their 95\% confidence intervals, and compared using the two-tailed $\mathrm{z}$ test. A $p$ value less than 0.05 indicates statistical significance.

\section{Results}

\section{Demographic information}

All survey replies are reported in Table 1.

A total of 2150 radiologist members of SIRM (amounting to $20.1 \%$ of members in good standing for the year 2020) joined the survey. The response rate for the demographics section of the survey was $100 \%(2150 / 2150)$.

The age (Question \#1) and gender (Question \#2) distributions of the survey respondents were comparable in all regions. Among respondents, 951 (44.2\%) worked in hot regions and the remaining 1199 (55.8\%) in other regions (Question \#3) (Fig. 1). More than 60\% of respondents worked at public facilities (Question \#4), with a higher proportion of private workers operating in hot regions [353/951 (37.1\%) vs 387/1199 (32.3\%), $p=0.0213$ ]. About half of respondents worked as staff radiologists (Question \#5), followed by private consultants, those latter being more densely distributed in hot regions [245/951 (25.7\%) vs $261 / 1199$ (21.8\%), $p=0.0343$ ].

\section{Personal and family impact of the COVID-19 outbreak}

Questions about the personal and family impact of the COVID-19 outbreak were answered by $96.6 \%$ (2076/2150) of survey participants.

More than $75 \%$ of respondents were partnered or married and slightly less than half had sons and/or daughters (Question \#6). About 70\% of all respondents had no known medical conditions other than possible COVID19 (Question \#7). About half of respondents were asymptomatic with negative testing (Question \#8), with $44 \%$ being asymptomatic without testing. Presence of COVID-19-related symptoms was more frequent in hot regions [48/912 (5.3\%) vs 20/1164 (1.7\%), $p<0.0001$ ].

More than $73 \%$ of respondents were afraid of passing on COVID-19 to their family members (Question \#9), and about two thirds had their family relationships moderately or severely affected by the crisis (Question \#10).

\section{Professional impact of the COVID-19 outbreak}

The response rate for the professional section of the survey was $91.7 \%$ (1972/2150). More than $85 \%$ of respondents worked more than $35 \mathrm{~h}$ a week (including guard and on-call shifts; Question \#11) before the crisis, and this proportion fell to less than $60 \%$ since that (Question \#12).

In hot regions, most (i.e. $>50 \%$ ) of the respondents' professional activity was dedicated to COVID-19 (Question \#13) in $242 / 865$ cases $(28.0 \%)$, more than in other regions 
Table 1 Distribution of answers in hot and other regions (see Supplement 1 for full list of answers).

\begin{tabular}{|c|c|c|c|c|}
\hline $\begin{array}{l}\text { Question } \\
\#\end{array}$ & Answer & $\begin{array}{l}\text { Rate of replies (hot } \\
\text { regions) } \\
951 / 2150 \\
\text { respondents } \\
(44.2 \%)\end{array}$ & $\begin{array}{l}\text { Rate of replies (other } \\
\text { regions) } \\
1199 / 2150 \\
\text { respondents }(55.8 \%)\end{array}$ & $\begin{array}{l}x^{2} \\
p \text { value }\end{array}$ \\
\hline 1 & $\begin{array}{l}\text { Under } 35 \text { years old } \\
36-65 \text { years old } \\
\text { Over } 65 \text { years old }\end{array}$ & $\begin{array}{l}178 / 951(18.7 \%) \\
649 / 951(68.2 \%) \\
124 / 951(13.0 \%)\end{array}$ & $\begin{array}{l}196 / 1199(16.3 \%) \\
849 / 1199(70.8 \%) \\
154 / 1199(12.8 \%)\end{array}$ & $\begin{array}{l}2.23 \\
(0.3279)\end{array}$ \\
\hline 2 & $\begin{array}{l}\text { Male } \\
\text { Female }\end{array}$ & $\begin{array}{l}469 / 951(49.3 \%) \\
482 / 951(50.7 \%)\end{array}$ & $\begin{array}{l}615 / 1199(51.3 \%) \\
584 / 1199(48.7 \%)\end{array}$ & $\begin{array}{l}0.75 \\
(0.3865)\end{array}$ \\
\hline 3 & $\begin{array}{l}\text { Hot regions (i.e. Emilia-Romagna, Lombardy, Piedmont, Veneto) } \\
\text { Other regions }\end{array}$ & $\begin{array}{l}951 / 951(100 \%) \\
0\end{array}$ & $\begin{array}{l}0 \\
1199 / 1199(100 \%)\end{array}$ & - \\
\hline 4 & $\begin{array}{l}\text { Public facility } \\
\text { Private facility accredited to the public health service } \\
\text { Private facility not accredited to the public health service } \\
\text { Retired private consultant }\end{array}$ & $\begin{array}{l}598 / 951(62.9 \%) \\
248 / 951(26.1 \%) \\
29 / 951(3.0 \%) \\
76 / 951(8.0 \%)\end{array}$ & $\begin{array}{l}812 / 1199(67.7 \%) \\
250 / 1199(20.9 \%) \\
47 / 1199(3.9 \%) \\
90 / 1199(7.5 \%)\end{array}$ & $\begin{array}{l}5.3 \\
(0.0213)^{*}\end{array}$ \\
\hline 5 & $\begin{array}{l}\text { Resident } \\
\text { Post-doc fellow, PhD student or outpatient specialist } \\
\text { Research fellow } \\
\text { Associate or full professor } \\
\text { Staff radiologist } \\
\text { Medical director } \\
\text { Private consultant }\end{array}$ & $\begin{array}{l}102 / 951(10.7 \%) \\
11 / 951(1.2 \%) \\
4 / 951(0.4 \%) \\
12 / 951(1.3 \%) \\
496 / 951(52.2 \%) \\
81 / 951(8.5 \%) \\
245 / 951(25.7 \%)\end{array}$ & $\begin{array}{l}118 / 1199(9.8 \%) \\
29 / 1199(2.4 \%) \\
14 / 1199(1.2 \%) \\
17 / 1199(1.4 \%) \\
631 / 1199(52.6 \%) \\
129 / 1199(10.8 \%) \\
261 / 1199(21.8 \%)\end{array}$ & $\begin{array}{l}4.48 \\
(0.0343)^{*}\end{array}$ \\
\hline $6^{\circ}$ & $\begin{array}{l}\text { Single } \\
\text { Partnered } \\
\text { Married } \\
\text { I have son(s) and/or daughter(s) } \\
\text { I have relatives other than son(s), daughter(s), partner and/or spouse }\end{array}$ & $\begin{array}{l}163 / 912(17.9 \%) \\
166 / 912(18.2 \%) \\
543 / 912(59.5 \%) \\
407 / 912(44.6 \%) \\
29 / 912(3.2 \%)\end{array}$ & $\begin{array}{l}190 / 1164(16.3 \%) \\
215 / 1164(18.5 \%) \\
700 / 1164(60.1 \%) \\
552 / 1164(47.4 \%) \\
45 / 1164(3.9 \%)\end{array}$ & $\begin{array}{l}0.76 \\
(0.3833)\end{array}$ \\
\hline $7^{\circ}$ & $\begin{array}{l}\text { None } \\
\text { Diabetes } \\
\text { Cardiovascular diseases } \\
\text { Respiratory diseases } \\
\text { Immunological disorders } \\
\text { Cancer } \\
\text { Anxiety } \\
\text { Depression } \\
\text { Other }\end{array}$ & $\begin{array}{l}634 / 912(69.5 \%) \\
24 / 912(2.6 \%) \\
121 / 912(13.3 \%) \\
40 / 912(4.4 \%) \\
6 / 912(0.7 \%) \\
40 / 912(4.4 \%) \\
32 / 912(3.5 \%) \\
16 / 912(1.7 \%) \\
82 / 912(9.0 \%)\end{array}$ & $\begin{array}{l}827 / 1164(71.0 \%) \\
26 / 1164(2.2 \%) \\
166 / 1164(14.3 \%) \\
43 / 1164(3.7 \%) \\
9 / 1164(0.8 \%) \\
26 / 1164(2.2 \%) \\
39 / 1164(3.3 \%) \\
17 / 1164(1.5 \%) \\
89 / 1164(7.6 \%)\end{array}$ & $\begin{array}{l}0.5 \\
(0.4795)\end{array}$ \\
\hline 8 & $\begin{array}{l}\text { Asymptomatic with negative testing } \\
\text { Asymptomatic with positive testing } \\
\text { Symptomatic with negative testing } \\
\text { Symptomatic with positive testing } \\
\text { Asymptomatic, no testing performed } \\
\text { Symptomatic, no testing performed }\end{array}$ & $\begin{array}{l}446 / 912(48.9 \%) \\
10 / 912(1.1 \%) \\
22 / 912(2.4 \%) \\
10 / 912(1.1 \%) \\
408 / 912(44.7 \%) \\
16 / 912(1.8 \%)\end{array}$ & $\begin{array}{l}622 / 1164(53.5 \%) \\
4 / 1164(0.3 \%) \\
8 / 1164(0.7 \%) \\
7 / 1164(0.6 \%) \\
518 / 1164(44.5 \%) \\
5 / 1164(0.4 \%)\end{array}$ & $\begin{array}{l}19.18 \\
(<0.0001)^{*}\end{array}$ \\
\hline 9 & $\begin{array}{l}\text { Yes } \\
\text { No }\end{array}$ & $\begin{array}{l}693 / 912(76.0 \%) \\
219 / 912(24.0 \%)\end{array}$ & $\begin{array}{l}853 / 1164(73.3 \%) \\
311 / 1164(26.7 \%)\end{array}$ & $\begin{array}{l}1.83 \\
(0.1761)\end{array}$ \\
\hline 10 & $\begin{array}{l}\text { No } \\
\text { Mildly } \\
\text { Moderately } \\
\text { Severely }\end{array}$ & $\begin{array}{l}107 / 912(11.7 \%) \\
206 / 912(22.6 \%) \\
326 / 912(35.8 \%) \\
273 / 912(29.9 \%)\end{array}$ & $\begin{array}{l}123 / 1164(10.6 \%) \\
299 / 1164(25.7 \%) \\
404 / 1164(34.7 \%) \\
338 / 1164(29.0 \%)\end{array}$ & $\begin{array}{l}0.75 \\
(0.3865)\end{array}$ \\
\hline 11 & $\begin{array}{l}\text { Less than } 35 \mathrm{~h} \\
36-50 \mathrm{~h} \\
51-65 \mathrm{~h} \\
\text { More than } 65 \mathrm{~h}\end{array}$ & $\begin{array}{l}108 / 865(12.5 \%) \\
613 / 865(70.9 \%) \\
128 / 865(14.8 \%) \\
16 / 865(1.8 \%)\end{array}$ & $\begin{array}{l}158 / 1107(14.3 \%) \\
789 / 1107(71.3 \%) \\
122 / 1107(11.0 \%) \\
38 / 1107(3.4 \%)\end{array}$ & $\begin{array}{l}1.18 \\
(0.2774)\end{array}$ \\
\hline 12 & $\begin{array}{l}\text { Less than } 35 \mathrm{~h} \\
36-50 \mathrm{~h} \\
51-65 \mathrm{~h} \\
\text { More than } 65 \mathrm{~h}\end{array}$ & $\begin{array}{l}351 / 865(40.6 \%) \\
435 / 865(50.3 \%) \\
71 / 865(8.2 \%) \\
8 / 865(0.9 \%)\end{array}$ & $\begin{array}{l}479 / 1107(43.3 \%) \\
558 / 1107(50.4 \%) \\
59 / 1107(5.3 \%) \\
11 / 1107(1.0 \%)\end{array}$ & $\begin{array}{l}1.34 \\
(0.2470)\end{array}$ \\
\hline 13 & $\begin{array}{l}0-25 \% \\
25-50 \% \\
50-75 \% \\
75-100 \%\end{array}$ & $\begin{array}{l}419 / 865(48.4 \%) \\
204 / 865(23.6 \%) \\
155 / 865(17.9 \%) \\
87 / 865(10.1 \%)\end{array}$ & $\begin{array}{l}689 / 1107(62.2 \%) \\
201 / 1107(18.2 \%) \\
135 / 1107(12.2 \%) \\
82 / 1107(7.4 \%)\end{array}$ & $\begin{array}{l}18.6 \\
(<0.0001)^{*}\end{array}$ \\
\hline
\end{tabular}


Table 1 (continued)

\begin{tabular}{|c|c|c|c|c|}
\hline $\begin{array}{l}\text { Question } \\
\#\end{array}$ & Answer & $\begin{array}{l}\text { Rate of replies (hot } \\
\text { regions) } \\
951 / 2150 \\
\text { respondents } \\
(44.2 \%)\end{array}$ & $\begin{array}{l}\text { Rate of replies (other } \\
\text { regions) } \\
1199 / 2150 \\
\text { respondents (55.8\%) }\end{array}$ & $\begin{array}{l}x^{2} \\
p \text { value }\end{array}$ \\
\hline $14^{\circ}$ & $\begin{array}{l}\text { I don't manage COVID-19 patients } \\
\text { Diagnosis of COVID-19 } \\
\text { Follow-up of COVID-19 patients } \\
\text { Severe forms and complications of COVID-19 } \\
\text { Other }\end{array}$ & $\begin{array}{l}196 / 865(22.7 \%) \\
546 / 865(63.1 \%) \\
482 / 865(55.7 \%) \\
154 / 865(17.8 \%) \\
94 / 865(10.9 \%)\end{array}$ & $\begin{array}{l}425 / 1107(38.4 \%) \\
560 / 1107(50.6 \%) \\
392 / 1107(35.4 \%) \\
115 / 1107(10.4 \%) \\
123 / 1107(11.1 \%)\end{array}$ & $\begin{array}{l}54.99 \\
(<0.0001)^{*}\end{array}$ \\
\hline 15 & $\begin{array}{l}\text { Never } \\
\text { Rarely } \\
\text { Quite often } \\
\text { Very often }\end{array}$ & $\begin{array}{l}229 / 865(26.5 \%) \\
345 / 865(39.9 \%) \\
259 / 865(29.9 \%) \\
32 / 865(3.7 \%)\end{array}$ & $\begin{array}{l}444 / 1107(40.1 \%) \\
454 / 1107(41.0 \%) \\
194 / 1107(17.5 \%) \\
15 / 1107(1.4 \%)\end{array}$ & $\begin{array}{l}55.13 \\
(<0.0001)^{*}\end{array}$ \\
\hline $16^{\circ}$ & $\begin{array}{l}\text { Emergency procedures } \\
\text { Oncologic imaging } \\
\text { Non-oncologic imaging } \\
\text { Urgent or non-deferrable interventional procedures } \\
\text { Elective interventional procedures } \\
\text { Other }\end{array}$ & $\begin{array}{l}625 / 865(72.2 \%) \\
614 / 865(71.0 \%) \\
406 / 865(46.9 \%) \\
130 / 865(15.0 \%) \\
30 / 865(3.5 \%) \\
109 / 865(12.6 \%)\end{array}$ & $\begin{array}{l}784 / 1107(70.8 \%) \\
730 / 1107(65.9 \%) \\
441 / 1107(39.8 \%) \\
123 / 1107(11.1 \%) \\
21 / 1107(1.9 \%) \\
168 / 1107(15.2 \%)\end{array}$ & $\begin{array}{l}0.42 \\
(0.5169)\end{array}$ \\
\hline 17 & $\begin{array}{l}\text { No } \\
\text { Mildly } \\
\text { Moderately } \\
\text { Severely }\end{array}$ & $\begin{array}{l}23 / 865(2.7 \%) \\
105 / 865(12.1 \%) \\
455 / 865(52.6 \%) \\
282 / 865(32.6 \%)\end{array}$ & $\begin{array}{l}39 / 1107(3.5 \%) \\
128 / 1107(11.6 \%) \\
592 / 1107(53.5 \%) \\
348 / 1107(31.4 \%)\end{array}$ & $\begin{array}{l}0.01 \\
(0.9203)\end{array}$ \\
\hline 18 & $\begin{array}{l}\text { No } \\
\text { Mildly } \\
\text { Moderately } \\
\text { Severely }\end{array}$ & $\begin{array}{l}62 / 865(7.2 \%) \\
117 / 865(13.5 \%) \\
350 / 865(40.5 \%) \\
336 / 865(38.8 \%)\end{array}$ & $\begin{array}{l}95 / 1107(8.6 \%) \\
166 / 1107(15.0 \%) \\
455 / 1107(41.1 \%) \\
391 / 1107(35.3 \%)\end{array}$ & $\begin{array}{l}2.17 \\
(0.1407)\end{array}$ \\
\hline 19 & $\begin{array}{l}\text { No } \\
\text { Mildly } \\
\text { Moderately } \\
\text { Severely }\end{array}$ & $\begin{array}{l}487 / 865(56.3 \%) \\
205 / 865(23.7 \%) \\
132 / 865(15.3 \%) \\
41 / 865(4.7 \%)\end{array}$ & $\begin{array}{l}647 / 1107(58.4 \%) \\
208 / 1107(18.8 \%) \\
180 / 1107(16.3 \%) \\
72 / 1107(6.5 \%)\end{array}$ & $\begin{array}{l}2.03 \\
(0.1542)\end{array}$ \\
\hline 20 & $\begin{array}{l}\text { No } \\
\text { Yes, they have improved } \\
\text { Yes, they have worsened } \\
\text { I hardly see my colleagues any more }\end{array}$ & $\begin{array}{l}497 / 865(57.5 \%) \\
117 / 865(13.5 \%) \\
108 / 865(12.5 \%) \\
143 / 865(16.5 \%)\end{array}$ & $\begin{array}{l}648 / 1107(58.5 \%) \\
115 / 1107(10.4 \%) \\
146 / 1107(13.2 \%) \\
198 / 1107(17.9 \%)\end{array}$ & $\begin{array}{l}0.88 \\
(0.3482)\end{array}$ \\
\hline 21 & $\begin{array}{l}\text { None } \\
\text { Less than 10\% } \\
10-30 \% \\
\text { More than } 30 \%\end{array}$ & $\begin{array}{l}278 / 865(32.1 \%) \\
482 / 865(55.7 \%) \\
96 / 865(11.1 \%) \\
9 / 865(1.1 \%)\end{array}$ & $\begin{array}{l}624 / 1107(56.4 \%) \\
447 / 1107(40.4 \%) \\
32 / 1107(2.9 \%) \\
4 / 1107(0.3 \%)\end{array}$ & $\begin{array}{l}56.43 \\
(<0.0001)^{*}\end{array}$ \\
\hline 22 & $\begin{array}{l}0-25 \% \\
25-50 \% \\
50-75 \% \\
75-100 \%\end{array}$ & $\begin{array}{l}346 / 865(40.0 \%) \\
269 / 865(31.1 \%) \\
158 / 865(18.3 \%) \\
92 / 865(10.6 \%)\end{array}$ & $\begin{array}{l}508 / 1107(45.9 \%) \\
326 / 1107(29.4 \%) \\
170 / 1107(15.4 \%) \\
103 / 1107(9.3 \%)\end{array}$ & $\begin{array}{l}4.27 \\
(0.0388)^{*}\end{array}$ \\
\hline 23 & $\begin{array}{l}\text { No } \\
\text { Mildly } \\
\text { Moderately } \\
\text { Severely }\end{array}$ & $\begin{array}{l}145 / 865(16.8 \%) \\
226 / 865(26.1 \%) \\
345 / 865(39.9 \%) \\
149 / 865(17.2 \%)\end{array}$ & $\begin{array}{l}171 / 1107(15.5 \%) \\
318 / 1107(28.7 \%) \\
443 / 1107(40.0 \%) \\
175 / 1107(15.8 \%)\end{array}$ & $\begin{array}{l}0.28 \\
(0.5967)\end{array}$ \\
\hline 24 & $\begin{array}{l}\text { Yes } \\
\text { No }\end{array}$ & $\begin{array}{l}569 / 865(65.8 \%) \\
296 / 865(34.2 \%)\end{array}$ & $\begin{array}{l}710 / 1107(64.1 \%) \\
397 / 1107(35.9 \%)\end{array}$ & $\begin{array}{l}0.51 \\
(0.4751)\end{array}$ \\
\hline 25 & $\begin{array}{l}\text { Yes } \\
\text { No }\end{array}$ & $\begin{array}{l}806 / 865(93.2 \%) \\
59 / 865(6.8 \%)\end{array}$ & $\begin{array}{l}996 / 1107(90.0 \%) \\
111 / 1107(10.0 \%)\end{array}$ & $\begin{array}{l}5.94 \\
(0.0148)^{*}\end{array}$ \\
\hline 26 & $\begin{array}{l}0-25 \% \\
25-50 \% \\
50-75 \% \\
75-100 \%\end{array}$ & $\begin{array}{l}63 / 856(7.4 \%) \\
270 / 856(31.5 \%) \\
372 / 856(43.5 \%) \\
151 / 856(17.6 \%)\end{array}$ & $\begin{array}{l}84 / 1095(7.7 \%) \\
352 / 1095(32.1 \%) \\
475 / 1095(43.4 \%) \\
184 / 1095(16.8 \%)\end{array}$ & $\begin{array}{l}0.13 \\
(0.7184)\end{array}$ \\
\hline 27 & $\begin{array}{l}\text { Yes } \\
\text { No } \\
\text { Don't know }\end{array}$ & $\begin{array}{l}80 / 856(9.3 \%) \\
425 / 856(49.7 \%) \\
351 / 856(41.0 \%)\end{array}$ & $\begin{array}{l}96 / 1095(8.8 \%) \\
611 / 1095(55.8 \%) \\
388 / 1095(35.4 \%)\end{array}$ & $\begin{array}{l}1.04 \\
(0.3078)\end{array}$ \\
\hline
\end{tabular}


Table 1 (continued)

\begin{tabular}{|c|c|c|c|c|}
\hline $\begin{array}{l}\text { Question } \\
\#\end{array}$ & Answer & $\begin{array}{l}\text { Rate of replies (hot } \\
\text { regions) } \\
951 / 2150 \\
\text { respondents } \\
(44.2 \%)\end{array}$ & $\begin{array}{l}\text { Rate of replies (other } \\
\text { regions) } \\
1199 / 2150 \\
\text { respondents }(55.8 \%)\end{array}$ & $\begin{array}{l}x^{2} \\
p \text { value }\end{array}$ \\
\hline $28^{\circ}$ & $\begin{array}{l}\text { Hydroalcoholic gel } \\
\text { Masks } \\
\text { Visors, goggles and protective gowns } \\
\text { Other } \\
\text { No }\end{array}$ & $\begin{array}{l}203 / 856(23.7 \%) \\
436 / 856(50.9 \%) \\
442 / 856(51.6 \%) \\
45 / 856(5.3 \%) \\
302 / 856(35.3 \%)\end{array}$ & $\begin{array}{l}296 / 1095(27.0 \%) \\
627 / 1095(57.3 \%) \\
655 / 1095(59.8 \%) \\
64 / 1095(5.8 \%) \\
308 / 1095(28.1 \%)\end{array}$ & $\begin{array}{l}11.11 \\
(0.0009)^{*}\end{array}$ \\
\hline 29 & $\begin{array}{l}\text { Yes (symptomatic) } \\
\text { Yes (higher risk) } \\
\text { Yes (psychiatric conditions) } \\
\text { No }\end{array}$ & $\begin{array}{l}35 / 856(4.1 \%) \\
16 / 856(1.9 \%) \\
13 / 856(1.5 \%) \\
792 / 856(92.5 \%)\end{array}$ & $\begin{array}{l}13 / 1095(1.2 \%) \\
31 / 1095(2.8 \%) \\
13 / 1095(1.2 \%) \\
1038 / 1095(94.8 \%)\end{array}$ & $\begin{array}{l}3.88 \\
(0.0489)^{*}\end{array}$ \\
\hline 30 & $\begin{array}{l}\text { No } \\
\text { Mildly } \\
\text { Moderately } \\
\text { Severely }\end{array}$ & $\begin{array}{l}225 / 856(26.3 \%) \\
269 / 856(31.4 \%) \\
280 / 856(32.7 \%) \\
82 / 856(9.6 \%)\end{array}$ & $\begin{array}{l}258 / 1095(23.6 \%) \\
378 / 1095(34.5 \%) \\
361 / 1095(33.0 \%) \\
98 / 1095(8.9 \%)\end{array}$ & $\begin{array}{l}0.01 \\
(0.9203)\end{array}$ \\
\hline 31 & $\begin{array}{l}\text { Yes } \\
\text { No }\end{array}$ & $\begin{array}{l}30 / 827(3.6 \%) \\
797 / 827(96.4 \%)\end{array}$ & $\begin{array}{l}24 / 1070(2.2 \%) \\
1046 / 1070(97.8 \%)\end{array}$ & $\begin{array}{l}2.75 \\
(0.0973)\end{array}$ \\
\hline $32^{\circ}$ & $\begin{array}{l}\text { Alcohol } \\
\text { Tobacco } \\
\text { Other } \\
\text { None }\end{array}$ & $\begin{array}{l}65 / 827(7.9 \%) \\
46 / 827(5.6 \%) \\
22 / 827(2.7 \%) \\
716 / 827(86.6 \%)\end{array}$ & $\begin{array}{l}50 / 1070(4.7 \%) \\
48 / 1070(4.5 \%) \\
33 / 1070(3.1 \%) \\
954 / 1070(89.2 \%)\end{array}$ & $\begin{array}{l}2.71 \\
(0.0997)\end{array}$ \\
\hline 33 & $\begin{array}{l}\text { No } \\
\text { Mildly } \\
\text { Moderately } \\
\text { Severely }\end{array}$ & $\begin{array}{l}336 / 827(40.6 \%) \\
286 / 827(34.6 \%) \\
157 / 827(19.0 \%) \\
48 / 827(5.8 \%)\end{array}$ & $\begin{array}{l}456 / 1070(42.6 \%) \\
352 / 1070(32.9 \%) \\
210 / 1070(19.6 \%) \\
52 / 1070(4.9 \%)\end{array}$ & $\begin{array}{l}0.01 \\
(0.9203)\end{array}$ \\
\hline 34 & $\begin{array}{l}\text { No } \\
\text { Mildly } \\
\text { Moderately } \\
\text { Severely }\end{array}$ & $\begin{array}{l}433 / 827(52.4 \%) \\
212 / 827(25.6 \%) \\
153 / 827(18.5 \%) \\
29 / 827(3.5 \%)\end{array}$ & $\begin{array}{l}556 / 1070(52.0 \%) \\
302 / 1070(28.2 \%) \\
186 / 1070(17.4 \%) \\
26 / 1070(2.4 \%)\end{array}$ & $\begin{array}{l}1.23 \\
(0.2674)\end{array}$ \\
\hline 35 & $\begin{array}{l}\text { Never } \\
\text { Rarely } \\
\text { Quite often } \\
\text { Very often }\end{array}$ & $\begin{array}{l}113 / 827(13.7 \%) \\
449 / 827(54.3 \%) \\
231 / 827(27.9 \%) \\
34 / 827(4.1 \%)\end{array}$ & $\begin{array}{l}179 / 1070(16.7 \%) \\
598 / 1070(55.9 \%) \\
261 / 1070(24.4 \%) \\
32 / 1070(3.0 \%)\end{array}$ & $\begin{array}{l}4.66 \\
(0.0309)^{*}\end{array}$ \\
\hline 36 & $\begin{array}{l}\text { Never } \\
\text { Rarely } \\
\text { Quite often } \\
\text { Very often }\end{array}$ & $\begin{array}{l}10 / 827(1.2 \%) \\
399 / 827(48.3 \%) \\
388 / 827(46.9 \%) \\
30 / 827(3.6 \%)\end{array}$ & $\begin{array}{l}14 / 1070(1.3 \%) \\
511 / 1070(47.8 \%) \\
506 / 1070(47.3 \%) \\
39 / 1070(3.6 \%)\end{array}$ & $\begin{array}{l}0.01 \\
(0.9203)\end{array}$ \\
\hline 37 & $\begin{array}{l}\text { Never } \\
\text { Rarely } \\
\text { Quite often } \\
\text { Very often }\end{array}$ & $\begin{array}{l}210 / 827(25.4 \%) \\
345 / 827(41.7 \%) \\
229 / 827(27.7 \%) \\
43 / 827(5.2 \%)\end{array}$ & $\begin{array}{l}280 / 1070(26.2 \%) \\
411 / 1070(38.4 \%) \\
332 / 1070(31.0 \%) \\
47 / 1070(4.4 \%)\end{array}$ & $\begin{array}{l}1.22 \\
(0.2694)\end{array}$ \\
\hline 38 & $\begin{array}{l}\text { Never } \\
\text { Rarely } \\
\text { Quite often } \\
\text { Very often }\end{array}$ & $\begin{array}{l}113 / 827(13.6 \%) \\
362 / 827(43.8 \%) \\
320 / 827(38.7 \%) \\
32 / 827(3.9 \%)\end{array}$ & $\begin{array}{l}134 / 1070(12.5 \%) \\
530 / 1070(49.5 \%) \\
361 / 1070(33.8 \%) \\
45 / 1070(4.2 \%)\end{array}$ & $\begin{array}{l}3.96 \\
(0.0466)^{*}\end{array}$ \\
\hline 39 & $\begin{array}{l}\text { More } \\
\text { Less } \\
\text { Nothing has changed }\end{array}$ & $\begin{array}{l}311 / 827(37.6 \%) \\
260 / 827(31.4 \%) \\
256 / 827(31.0 \%)\end{array}$ & $\begin{array}{l}397 / 1070(37.1 \%) \\
354 / 1070(33.1 \%) \\
319 / 1070(29.8 \%)\end{array}$ & $\begin{array}{l}0.5 \\
(0.4795)\end{array}$ \\
\hline 40 & $\begin{array}{l}\text { Never } \\
\text { Rarely } \\
\text { Quite often } \\
\text { Very often }\end{array}$ & $\begin{array}{l}327 / 827(39.5 \%) \\
372 / 827(45.0 \%) \\
113 / 827(13.7 \%) \\
15 / 827(1.8 \%)\end{array}$ & $\begin{array}{l}412 / 1070(38.5 \%) \\
491 / 1070(45.9 \%) \\
150 / 1070(14.0 \%) \\
17 / 1070(1.6 \%)\end{array}$ & $\begin{array}{l}0 \\
(1)\end{array}$ \\
\hline 41 & $\begin{array}{l}\text { Never } \\
\text { Rarely } \\
\text { Quite often } \\
\text { Very often }\end{array}$ & $\begin{array}{l}593 / 827(71.7 \%) \\
192 / 827(23.2 \%) \\
37 / 827(4.5 \%) \\
5 / 827(0.6 \%)\end{array}$ & $\begin{array}{l}762 / 1070(71.2 \%) \\
250 / 1070(23.4 \%) \\
46 / 1070(4.3 \%) \\
12 / 1070(1.1 \%)\end{array}$ & $\begin{array}{l}0.05 \\
(0.8231)\end{array}$ \\
\hline 42 & $\begin{array}{l}\text { More } \\
\text { Less } \\
\text { Nothing has changed }\end{array}$ & $\begin{array}{l}34 / 827(4.1 \%) \\
524 / 827(63.4 \%) \\
269 / 827(32.5 \%)\end{array}$ & $\begin{array}{l}51 / 1070(4.8 \%) \\
627 / 1070(58.6 \%) \\
392 / 1070(36.6 \%)\end{array}$ & $\begin{array}{l}3.29 \\
(0.0697)\end{array}$ \\
\hline
\end{tabular}


Table 1 (continued)

${ }^{\circ}$ Multiple answers allowed. ${ }^{*} p<0.05$

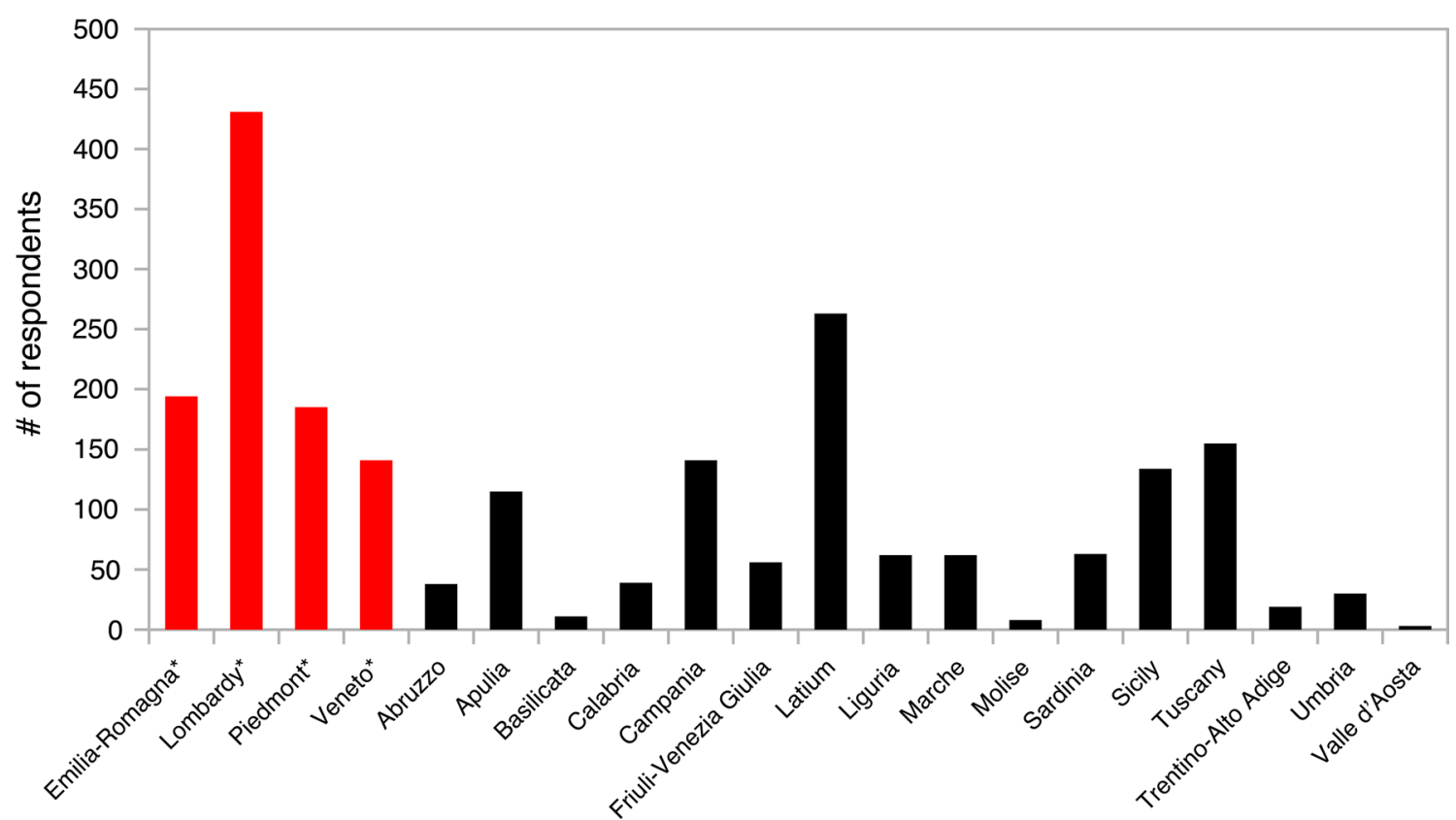

Fig. 1 Bar chart showing the distribution of survey respondents in hot regions (asterisk, left hand side of the chart) and in other regions (right hand side)

[217/1107 cases $(19.6 \%), p<0.0001]$. The proportion of respondents who did not manage COVID-19 patients (Question \#14) was lower in hot regions [196/865 (22.7\%) vs $425 / 1107(38.4 \%), p<0.0001$ ], and more procedures for severe forms and complications of COVID-19 were performed in hot regions. Incidental diagnoses of COVID19 (Question \#15) were made more frequently in hot regions [291/865 (33.6\%) vs 209/1107 (18.9\%), $p<0.0001]$.

The most frequent procedures performed on nonCOVID-19 patients during the crisis (Question \#16) included emergency imaging (around 70\%), followed by oncologic imaging (about two thirds of respondents). About $85 \%$ of respondents thought that the crisis could have a moderately or severely negative impact on the management of non-COVID-19 patients (Question \#17), and more than $75 \%$ were moderately or severely concerned that a work overload will occur after the crisis to catch up on deferred procedures (Question \#18).

Only about $20 \%$ respondents believed that the crisis had moderately or severely affected their radiological training and/or skills (Question \#19), but this proportion was much higher among residents [58/86 (67.4\%) from hot regions and 82/109 (75.2\%) from other regions; $p=0.2987]$. The majority of respondents denied any relevant impact of the crisis on their relationships with colleagues (Question \#20). The respondents' colleagues in hot regions had been infected more frequently than in other regions [Question \#21; 105/865 (12.1\%) vs $36 / 1107$ (3.3\%) of more than $10 \%$ of infected colleagues, $p<0.0001]$. Respondents were more afraid of getting infected at work [Question \#22; 250/865 (28.9\%) vs $273 / 1107(24.7 \%)$ over a 50\% threshold, $p=0.0388$ ], with male respondents from hot regions being more afraid compared to those from other regions [ $\log$ OR 0.4049 (CI95\% 0.1071 $\div 0.7027$ ), $p=0.0077$ ] (Table 2).

More than $55 \%$ of respondents had their emotional stress at work moderately or severely increased (Question \#23). Most respondents also felt that their professional contribution could be relevant (Question \#24), and that workplaces will need to be updated after the crisis [Question \#25; 806/865 (93.2\%) in hot regions vs 996/1107 (90.0\%) in other regions, $p=0.0148]$.

\section{Socioeconomic impact of the COVID-19 outbreak}

The response rate for the socioeconomic section of the survey was $90.7 \%$ (1951/2150). More than $60 \%$ of respondents estimated that the crisis led to a workload reduction higher than $50 \%$ (Question \#26). This figure 
Table 2 Distribution of answers in hot and other regions stratified by respondents' gender.

\begin{tabular}{|c|c|c|c|c|}
\hline Question \# & $\begin{array}{l}\% \text { of replies } \\
\text { (hot regions) }\end{array}$ & $\begin{array}{l}\% \text { of replies } \\
\text { (other regions) }\end{array}$ & $\log$ OR (Cl95\%) & $z$ (p value) \\
\hline 9 & $\begin{array}{l}\text { Male: } 314 / 452(69.5 \%) \\
\text { Female: } 379 / 460(82.4 \%)\end{array}$ & $\begin{array}{l}\text { Male: 399/598 (66.7\%) } \\
\text { Female: 454/566 (80.2\%) }\end{array}$ & $\begin{array}{l}\text { Male: } 0.1265(-0.1860 \div 0.4389) \\
\text { Female: } 0.1435(-0.1243 \div 0.4112)\end{array}$ & $\begin{array}{l}\text { Male: } 0.7934(0.4275) \\
\text { Female: } 1.0503(0.2936)\end{array}$ \\
\hline 10 & $\begin{array}{l}\text { Male: } 270 / 452(59.7 \%) \\
\text { Female: } 329 / 460(71.5 \%)\end{array}$ & $\begin{array}{l}\text { Male: } 321 / 598(53.7 \%) \\
\text { Female: } 421 / 566(74.4 \%)\end{array}$ & $\begin{array}{l}\text { Male: } 0.2470(-0.0293 \div 0.5233) \\
\text { Female: }-0.1450(-0.3929 \div 0.1029)\end{array}$ & $\begin{array}{l}\text { Male: } 1.7521(0.0798) \\
\text { Female: }-1.1467(0.2515)\end{array}$ \\
\hline 20 & $\begin{array}{l}\text { Male: } 116 / 429(27.0 \%) \\
\text { Female: } 135 / 436(31.0 \%)\end{array}$ & $\begin{array}{l}\text { Male: } 149 / 569(26.2 \%) \\
\text { Female: } 195 / 538(36.2 \%)\end{array}$ & $\begin{array}{l}\text { Male: } 0.0437(-0.2506 \div 0.3380) \\
\text { Female: }-0.2371(-0.4937 \div 0.0195)\end{array}$ & $\begin{array}{l}\text { Male: } 0.2910(0.7710) \\
\text { Female: }-1.8113(0.0701)\end{array}$ \\
\hline 22 & $\begin{array}{l}\text { Male: } 102 / 429(23.8 \%) \\
\text { Female: } 148 / 436(33.9 \%)\end{array}$ & $\begin{array}{l}\text { Male: } 98 / 569(17.2 \%) \\
\text { Female: } 175 / 538(32.5 \%)\end{array}$ & $\begin{array}{l}\text { Male: } 0.4049(0.1071 \div 0.7027) \\
\text { Female: } 0.0639(-0.2188 \div 0.3465)\end{array}$ & $\begin{array}{l}\text { Male: } 2.6646(0.0077)^{*} \\
\text { Female: } 0.4429(0.6578)\end{array}$ \\
\hline 23 & $\begin{array}{l}\text { Male: 200/429 (46.6\%) } \\
\text { Female: } 294 / 436(67.4 \%)\end{array}$ & $\begin{array}{l}\text { Male: } 270 / 569(47.5 \%) \\
\text { Female: 348/538 (64.7\%) }\end{array}$ & $\begin{array}{l}\text { Male: }-0.0334(-0.3093 \div 0.2425) \\
\text { Female: } 0.1226(-0.1190 \div 0.3641)\end{array}$ & $\begin{array}{l}\text { Male: }-0.2372(0.8125) \\
\text { Female: } 0.9947(0.3199)\end{array}$ \\
\hline 31 & $\begin{array}{l}\text { Male: } 11 / 406(2.7 \%) \\
\text { Female: } 19 / 421(4.5 \%)\end{array}$ & $\begin{array}{l}\text { Male: } 11 / 549(2.0 \%) \\
\text { Female: } 13 / 521(2.5 \%)\end{array}$ & $\begin{array}{l}\text { Male: } 0.3090(-0.4465 \div 1.0644) \\
\text { Female: } 0.6135(-0.1985 \div 1.4256)\end{array}$ & $\begin{array}{l}\text { Male: } 0.8016(0.4228) \\
\text { Female: } 1.4808(0.1387)\end{array}$ \\
\hline 32 & $\begin{array}{l}\text { Male: 59/406 (14.5\%) } \\
\text { Female: 52/421 (12.4\%) }\end{array}$ & $\begin{array}{l}\text { Male: } 61 / 549(11.1 \%) \\
\text { Female: 55/521 (10.6\%) }\end{array}$ & $\begin{array}{l}\text { Male: } 0.3077(-0.0929 \div 0.7082) \\
\text { Female: } 0.1773(-0.2086 \div 0.5632)\end{array}$ & $\begin{array}{l}\text { Male: } 1.5053(0.1322) \\
\text { Female: } 0.9004(0.3679)\end{array}$ \\
\hline 33 & $\begin{array}{l}\text { Male: 80/406 (19.7\%) } \\
\text { Female: 125/421 (29.7\%) }\end{array}$ & $\begin{array}{l}\text { Male: 109/549 (19.9\%) } \\
\text { Female: } 153 / 521(29.4 \%)\end{array}$ & $\begin{array}{l}\text { Male: }-0.0094(-0.3312 \div 0.3123) \\
\text { Female: } 0.0156(-0.2664 \div 0.2976)\end{array}$ & $\begin{array}{l}\text { Male: }-0.0575(0.9541) \\
\text { Female: } 0.1084(0.9137)\end{array}$ \\
\hline 34 & $\begin{array}{l}\text { Male: } 74 / 406(18.2 \%) \\
\text { Female: 108/421 (25.7\%) }\end{array}$ & $\begin{array}{l}\text { Male: } 96 / 549(17.5 \%) \\
\text { Female: } 116 / 521(22.3 \%)\end{array}$ & $\begin{array}{l}\text { Male: } 0.0505(-0.2832 \div 0.3841) \\
\text { Female: } 0.1862(-0.1156 \div 0.4881)\end{array}$ & $\begin{array}{l}\text { Male: } 0.2965(0.7668) \\
\text { Female: } 1.2093(0.2265)\end{array}$ \\
\hline 35 & $\begin{array}{l}\text { Male: } 102 / 406(25.1 \%) \\
\text { Female: } 163 / 421(38.7 \%)\end{array}$ & $\begin{array}{l}\text { Male: } 120 / 549(21.9 \%) \\
\text { Female: } 173 / 521(33.2 \%)\end{array}$ & $\begin{array}{l}\text { Male: } 0.1819(-0.1160 \div 0.4798) \\
\text { Female: } 0.2397(-0.0327 \div 0.5121)\end{array}$ & $\begin{array}{l}\text { Male: } 1.1968(0.2314) \\
\text { Female: } 1.7246(0.0846)\end{array}$ \\
\hline 36 & $\begin{array}{l}\text { Male: 209/406 (51.5\%) } \\
\text { Female: 209/421 (49.6\%) }\end{array}$ & $\begin{array}{l}\text { Male: } 285 / 549(51.9 \%) \\
\text { Female: } 260 / 521(49.9 \%)\end{array}$ & $\begin{array}{l}\text { Male: }-0.0174(-0.2901 \div 0.2553) \\
\text { Female: }-0.0104(-0.2503 \div 0.2294)\end{array}$ & $\begin{array}{l}\text { Male: }-0.1251(0.9004) \\
\text { Female: }-0.0851(0.9322)\end{array}$ \\
\hline 37 & $\begin{array}{l}\text { Male: } 116 / 406(28.6 \%) \\
\text { Female: } 156 / 421(37.1 \%)\end{array}$ & $\begin{array}{l}\text { Male: } 150 / 549(27.3 \%) \\
\text { Female: } 229 / 521(44.0 \%)\end{array}$ & $\begin{array}{l}\text { Male: } 0.0620(-0.2303 \div 0.3544) \\
\text { Female: }-0.2868(-0.5421 \div-0.0316)\end{array}$ & $\begin{array}{l}\text { Male: } 0.4159(0.6775) \\
\text { Female: }-2.2023(0.0276)^{*}\end{array}$ \\
\hline 38 & $\begin{array}{l}\text { Male: } 137 / 406(33.7 \%) \\
\text { Female: } 215 / 421(51.1 \%)\end{array}$ & $\begin{array}{l}\text { Male: } 160 / 549(29.1 \%) \\
\text { Female: } 246 / 521(47.2 \%)\end{array}$ & $\begin{array}{l}\text { Male: } 0.2137(-0.0671 \div 0.4945) \\
\text { Female: } 0.1542(-0.0977 \div 0.4061)\end{array}$ & $\begin{array}{l}\text { Male: } 1.4916(0.1358) \\
\text { Female: } 1.1997(0.2303)\end{array}$ \\
\hline 39 & $\begin{array}{l}\text { Male: } 103 / 406(25.4 \%) \\
\text { Female: } 157 / 421(37.3 \%)\end{array}$ & $\begin{array}{l}\text { Male: } 156 / 549(28.4 \%) \\
\text { Female: } 198 / 521(38.0 \%)\end{array}$ & $\begin{array}{l}\text { Male: }-0.1551(-0.4534 \div 0.1433) \\
\text { Female: }-0.0303(-0.2866 \div 0.2260)\end{array}$ & $\begin{array}{l}\text { Male: }-1.0187(0.3083) \\
\text { Female: }-0.2318(0.8167)\end{array}$ \\
\hline 40 & $\begin{array}{l}\text { Male: } 41 / 406(10.1 \%) \\
\text { Female: } 87 / 421(20.7 \%)\end{array}$ & $\begin{array}{l}\text { Male: } 55 / 549(10.0 \%) \\
\text { Female: } 112 / 521(21.5 \%)\end{array}$ & $\begin{array}{l}\text { Male: } 0.0089(-0.3910 \div 0.4087) \\
\text { Female: }-0.0500(-0.3983 \div 0.2983)\end{array}$ & $\begin{array}{l}\text { Male: } 0.0435(0.9653) \\
\text { Female: }-0.2815(0.7783)\end{array}$ \\
\hline 41 & $\begin{array}{l}\text { Male: } 14 / 406(3.4 \%) \\
\text { Female: } 28 / 421(6.7 \%)\end{array}$ & $\begin{array}{l}\text { Male: } 17 / 549(3.1 \%) \\
\text { Female: } 41 / 521(7.9 \%)\end{array}$ & $\begin{array}{l}\text { Male: } 0.1112(-0.5454 \div 0.7679) \\
\text { Female: }-0.1814(-0.7601 \div 0.3973)\end{array}$ & $\begin{array}{l}\text { Male: } 0.3320(0.7399) \\
\text { Female: }-0.6143(0.5390)\end{array}$ \\
\hline 42 & $\begin{array}{l}\text { Male: } 88 / 406(21.7 \%) \\
\text { Female: } 181 / 421(43.0 \%)\end{array}$ & $\begin{array}{l}\text { Male: } 142 / 549(25.9 \%) \\
\text { Female: } 250 / 521(48.0 \%)\end{array}$ & $\begin{array}{l}\text { Male: }-0.2317(-0.5366 \div 0.0732) \\
\text { Female: }-0.2015(-0.4585 \div 0.0555)\end{array}$ & $\begin{array}{l}\text { Male: }-1.4896(0.1363) \\
\text { Female: }-1.5368(0.1243)\end{array}$ \\
\hline
\end{tabular}

${ }^{*} p<0.05$

was higher for private than for public workers $[224 / 308$ $(72.7 \%)$ in hot regions vs $226 / 340(66.5 \%)$ in other regions; $p=0.1010]$.

Less than $10 \%$ of respondents thought that the private sector had been adequately protected during the crisis (Question \#27). The majority of respondents had difficulty obtaining personal protective equipment (PPE) (Question \#28), and this percentage was lower in hot regions $(64.7 \%$ vs $71.9 \%, p=0.0009)$.

More than $90 \%$ of respondents were not forced to stay away from work (Question \#29), but the percentage of those who did was higher in hot regions [64/856 $(7.5 \%)$ vs $57 / 1095$ (5.2\%), $p=0.0489$ ]. More than $40 \%$ of respondents were moderately or severely worried that the crisis will have a detrimental impact on their and their colleagues' professional activity (Question \#30).

\section{Psychological impact of the COVID-19 outbreak}

The response rate for the psychological section of the survey was $88.2 \%$ (1897/2150). The majority of respondents did not start any psychiatric treatment (Question $\# 31$ ), and the use of recreational substances did not change during the crisis (Question \#32).

About $40 \%$ of radiologists denied suffering from sleep disturbances during the crisis (Question \#33), with moderate or severe disturbances being reported by about one quarter of respondents. Sleep disturbances mostly had a mild or no impact on daily functioning (Question \#34), 
although sleep was moderately or severely impaired in about $20 \%$ of respondents.

A consistent minority of respondents had negative thoughts quite often or very often during the crisis (Question \#35), and more so in hot regions [265/827 (32.0\%) vs $293 / 1070$ (27.4\%), $p=0.0309]$. Such likelihood was also increased in single-living respondents from hot regions than from other regions $[\log \mathrm{OR}=0.7108$ (CI95\% $0.3445 \div 1.0770$ ), $p=0.0001$ ] (Table 3 ).

The percentage of respondents being in a good mood was comparable to those who were not (Question \#36). However, more than $30 \%$ of respondents felt quite often or very often like living in slow motion (Question \#37), with females from hot regions being less susceptible than those from other regions $[\log$ OR -0.2868 (CI95\% $-0.5421 \div-0.0316), p=0.0276]$. More respondents from hot regions than from other regions felt restless or nervous quite often or very often (Question \#38) [352/827 (42.6\%) vs 406/1070 (37.9\%), $p=0.0466$ ].

About $30 \%$ of respondents enjoyed relaxing and doing the same things as before the crisis (Question \#39). A relatively small proportion of respondents complained about having quite often or very often feelings of fear (Question \#40, about 15\%) or panic (Question \#41, about $5 \%$ ). About $60 \%$ of respondents took less care of their physical appearance during the crisis (Question \#42).

\section{Discussion}

To our knowledge, this is the first survey by a European national radiological society to assess the impact of the COVID-19 pandemic on its members' overall wellbeing.

Table 3 Distribution of answers in hot and other regions stratified by respondents' living condition (i.e. single or not).

\begin{tabular}{|c|c|c|c|c|}
\hline Question \# & $\begin{array}{l}\% \text { of replies } \\
\text { (hot regions) }\end{array}$ & $\begin{array}{l}\% \text { of replies } \\
\text { (other regions) }\end{array}$ & $\log$ OR (C195\%) & z (p value) \\
\hline 9 & $\begin{array}{l}\text { Single: } 115 / 163(70.6 \%) \\
\text { Others: } 578 / 749(77.2 \%)\end{array}$ & $\begin{array}{l}\text { Single: } 141 / 190(74.2 \%) \\
\text { Others: } 712 / 974(73.1 \%)\end{array}$ & $\begin{array}{l}\text { Single: }-0.1832(-0.5608 \div 0.1944) \\
\text { Others: } 0.2182(-0.1364 \div 0.5727)\end{array}$ & $\begin{array}{l}\text { Single: }-0.9511(0.3415) \\
\text { Others: } 1.2061(0.2278)\end{array}$ \\
\hline 10 & $\begin{array}{l}\text { Single: } 124 / 163(76.1 \%) \\
\text { Others: } 475 / 749(63.4 \%)\end{array}$ & $\begin{array}{l}\text { Single: } 143 / 190(75.3 \%) \\
\text { Others: } 599 / 974(61.5 \%)\end{array}$ & $\begin{array}{l}\text { Single: } 0.0440(-0.3453 \div 0.4334) \\
\text { Others: } 0.0819(-0.2721 \div 0.4358)\end{array}$ & $\begin{array}{l}\text { Single: } 0.2216(0.8246) \\
\text { Others: } 0.4533(0.6503)\end{array}$ \\
\hline 20 & $\begin{array}{l}\text { Single: } 56 / 155(36.1 \%) \\
\text { Others: } 195 / 710(27.5 \%)\end{array}$ & $\begin{array}{l}\text { Single: } 54 / 182(29.7 \%) \\
\text { Others: } 290 / 925(31.4 \%)\end{array}$ & $\begin{array}{l}\text { Single: } 0.2933(-0.0736 \div 0.6601) \\
\text { Others: }-0.1874(-0.5345 \div 0.1596)\end{array}$ & $\begin{array}{l}\text { Single: } 1.5670(0.1171) \\
\text { Others: }-1.0585(0.2898)\end{array}$ \\
\hline 22 & $\begin{array}{l}\text { Single: } 35 / 155(22.6 \%) \\
\text { Others: } 215 / 710(30.3 \%)\end{array}$ & $\begin{array}{l}\text { Single: } 36 / 182(19.8 \%) \\
\text { Others: } 237 / 925(25.6 \%)\end{array}$ & $\begin{array}{l}\text { Single: } 0.1679(-0.2412 \div 0.5771) \\
\text { Others: } 0.2318(-0.1617 \div 0.6253)\end{array}$ & $\begin{array}{l}\text { Single: } 0.8045(0.4211) \\
\text { Others: } 1.1547(0.2482)\end{array}$ \\
\hline 23 & $\begin{array}{l}\text { Single: } 83 / 155(53.5 \%) \\
\text { Others: } 411 / 710(57.9 \%)\end{array}$ & $\begin{array}{l}\text { Single: } 93 / 182(51.1 \%) \\
\text { Others: 525/925 (56.8\%) }\end{array}$ & $\begin{array}{l}\text { Single: } 0.0982(-0.2508 \div 0.4473) \\
\text { Others: } 0.0462(-0.2722 \div 0.3646)\end{array}$ & $\begin{array}{l}\text { Single: } 0.5515(0.5813) \\
\text { Others: } 0.2845(0.7760)\end{array}$ \\
\hline 31 & $\begin{array}{l}\text { Single: } 4 / 150(2.7 \%) \\
\text { Others: } 26 / 677(3.8 \%)\end{array}$ & $\begin{array}{l}\text { Single: } 3 / 178(1.7 \%) \\
\text { Others: } 21 / 892(2.4 \%)\end{array}$ & $\begin{array}{l}\text { Single: } 0.4689(-0.5990 \div 1.5367) \\
\text { Others: } 0.5047(-0.7159 \div 1.7253)\end{array}$ & $\begin{array}{l}\text { Single: } 0.8606(0.3895) \\
\text { Others: } 0.8105(0.4176)\end{array}$ \\
\hline 32 & $\begin{array}{l}\text { Single: } 23 / 150(15.3 \%) \\
\text { Others: } 88 / 677(13.0 \%)\end{array}$ & $\begin{array}{l}\text { Single: } 25 / 178(14.0 \%) \\
\text { Others: } 91 / 892(10.2 \%)\end{array}$ & $\begin{array}{l}\text { Single: } 0.1029(-0.3946 \div 0.6003) \\
\text { Others: } 0.2739(-0.2013 \div 0.7491)\end{array}$ & $\begin{array}{l}\text { Single: } 0.4053(0.6853) \\
\text { Others: } 1.1298(0.2586)\end{array}$ \\
\hline 33 & $\begin{array}{l}\text { Single: } 47 / 150(31.3 \%) \\
\text { Others: } 158 / 677(23.3 \%)\end{array}$ & $\begin{array}{l}\text { Single: } 49 / 178(27.5 \%) \\
\text { Others: } 213 / 892(23.9 \%)\end{array}$ & $\begin{array}{l}\text { Single: } 0.1834(-0.2049 \div 0.5717) \\
\text { Others: }-0.0300(-0.3931 \div 0.3332)\end{array}$ & $\begin{array}{l}\text { Single: } 0.9259(0.3545) \\
\text { Others: }-0.1618(0.8715)\end{array}$ \\
\hline 34 & $\begin{array}{l}\text { Single: } 40 / 150(26.7 \%) \\
\text { Others: } 142 / 677(21.0 \%)\end{array}$ & $\begin{array}{l}\text { Single: } 38 / 178(21.3 \%) \\
\text { Others: } 174 / 892(19.5 \%)\end{array}$ & $\begin{array}{l}\text { Single: } 0.2925(-0.1140 \div 0.6989) \\
\text { Others: } 0.0910(-0.3039 \div 0.4859)\end{array}$ & $\begin{array}{l}\text { Single: } 1.4103(0.1584) \\
\text { Others: } 0.4515(0.6516)\end{array}$ \\
\hline 35 & $\begin{array}{l}\text { Single: } 59 / 150(39.3 \%) \\
\text { Others: } 206 / 677(30.4 \%)\end{array}$ & $\begin{array}{l}\text { Single: } 43 / 178(24.2 \%) \\
\text { Others: } 250 / 892(28.0 \%)\end{array}$ & $\begin{array}{l}\text { Single: } 0.7108(0.3445 \div 1.0770) \\
\text { Others: } 0.1161(-0.2569 \div 0.4892)\end{array}$ & $\begin{array}{l}\text { Single: } 3.8037(0.0001)^{*} \\
\text { Others: } 0.6103(0.5417)\end{array}$ \\
\hline 36 & $\begin{array}{l}\text { Single: } 79 / 150(52.7 \%) \\
\text { Others: } 339 / 677(50.1 \%)\end{array}$ & $\begin{array}{l}\text { Single: } 89 / 178(50.0 \%) \\
\text { Others: } 456 / 892(51.1 \%)\end{array}$ & $\begin{array}{l}\text { Single: } 0.1068(-0.2474 \div 0.4609) \\
\text { Others: }-0.0419(-0.3637 \div 0.2799)\end{array}$ & $\begin{array}{l}\text { Single: } 0.5909(0.5546) \\
\text { Others: }-0.2552(0.7986)\end{array}$ \\
\hline 37 & $\begin{array}{l}\text { Single: } 52 / 150(34.7 \%) \\
\text { Others: } 220 / 677(32.5 \%)\end{array}$ & $\begin{array}{l}\text { Single: } 55 / 178(30.9 \%) \\
\text { Others: } 324 / 892(36.3 \%)\end{array}$ & $\begin{array}{l}\text { Single: } 0.1711(-0.2016 \div 0.5439) \\
\text { Others: }-0.1697(-0.5157 \div 0.1763)\end{array}$ & $\begin{array}{l}\text { Single: } 0.8998(0.3682) \\
\text { Others: }-0.9612(0.3364)\end{array}$ \\
\hline 38 & $\begin{array}{l}\text { Single: } 62 / 150(41.3 \%) \\
\text { Others: } 290 / 677(42.8 \%)\end{array}$ & $\begin{array}{l}\text { Single: } 63 / 178(35.4 \%) \\
\text { Others: } 343 / 892(38.5 \%)\end{array}$ & $\begin{array}{l}\text { Single: } 0.2516(-0.1073 \div 0.6105) \\
\text { Others: } 0.1818(-0.1537 \div 0.5174)\end{array}$ & $\begin{array}{l}\text { Single: } 1.3741(0.1694) \\
\text { Others: } 1.0621(0.2882)\end{array}$ \\
\hline 39 & $\begin{array}{l}\text { Single: } 51 / 150(34.0 \%) \\
\text { Others: } 209 / 677(30.9 \%)\end{array}$ & $\begin{array}{l}\text { Single: } 55 / 178(30.9 \%) \\
\text { Others: } 299 / 892(33.5 \%)\end{array}$ & $\begin{array}{l}\text { Single: } 0.1416(-0.2336 \div 0.5167) \\
\text { Others: }-0.1214(-0.4684 \div 0.2256)\end{array}$ & $\begin{array}{l}\text { Single: } 0.7396(0.4595) \\
\text { Others: }-0.6856(0.4930)\end{array}$ \\
\hline 40 & $\begin{array}{l}\text { Single: } 27 / 150(18.0 \%) \\
\text { Others: } 101 / 677(14.9 \%)\end{array}$ & $\begin{array}{l}\text { Single: } 26 / 178(14.6 \%) \\
\text { Others: } 141 / 892(15.8 \%)\end{array}$ & $\begin{array}{l}\text { Single: } 0.2494(-0.2177 \div 0.7166) \\
\text { Others: }-0.0683(-0.5215 \div 0.3849)\end{array}$ & $\begin{array}{l}\text { Single: } 1.0466(0.2953) \\
\text { Others: }-0.2956(0.7675)\end{array}$ \\
\hline 41 & $\begin{array}{l}\text { Single: } 10 / 150(6.7 \%) \\
\text { Others: } 32 / 677(4.7 \%)\end{array}$ & $\begin{array}{l}\text { Single: } 9 / 178(5.1 \%) \\
\text { Others: } 49 / 892(5.5 \%)\end{array}$ & $\begin{array}{l}\text { Single: } 0.2936(-0.4396 \div 1.0268) \\
\text { Others: }-0.1584(-0.8881 \div 0.5714)\end{array}$ & $\begin{array}{l}\text { Single: } 0.7849(0.4325) \\
\text { Others: }-0.4254(0.6705)\end{array}$ \\
\hline 42 & $\begin{array}{l}\text { Single: } 51 / 150(34.0 \%) \\
\text { Others: } 218 / 677(32.2 \%)\end{array}$ & $\begin{array}{l}\text { Single: } 64 / 178(36.0 \%) \\
\text { Others: } 328 / 892(36.8 \%)\end{array}$ & $\begin{array}{l}\text { Single: }-0.0860(-0.4603 \div 0.2883) \\
\text { Others: }-0.2025(-0.5375 \div 0.1325)\end{array}$ & $\begin{array}{l}\text { Single: }-0.4502(0.6526) \\
\text { Others: }-1.1847(0.2361)\end{array}$ \\
\hline
\end{tabular}


Our survey has revealed a sensible impact of the COVID-19 outbreak on radiologists' life, with differences in specific items occurring in hot regions. The majority of respondents were afraid of spreading the infection and felt that the crisis had affected their family relationships and will damage their own and their colleagues' professional activity. Such concerns were independent of the severity of the crisis on a per-region basis, suggesting a generalized discomfort and emotional distress, as also highlighted by answers to the psychological part of the survey.

However, radiologists from hot regions were understandably more afraid of getting infected at work and were also more exposed than those from other regions in terms of a more intensive management of COVID-19 patients, more incidental diagnoses of COVID-19, and a higher proportion of infected colleagues. In a Chinese survey on mental health outcomes of 1257 healthcare professionals (of whom 60\% operating in Wuhan), psychological distress was more common in frontline healthcare workers engaged in direct diagnosis, treatment and care of COVID-19 patients, with participants from outside Hubei province being at a lower risk of experiencing distress than those in Wuhan [16]. In another Chinese survey among radiology departments in 32 public hospitals, respondents showed an overall low toughness dimension score, along with a negative correlation between respondents' perceived stress and resilience. This survey also showed that knowledge of COVID-19, knowledge of COVID-19 protective measures and availability of adequate protective materials were independent influencing factors for resilience [17]. Although more than half of our survey respondents had difficulty sourcing PPE, such difficulty was higher in non-hot regions, which might have been less prepared to the crisis as hot regions experienced its peak with some time advance. This should be taken into account for planning the activity of radiology departments during the post-shutdown phase of the COVID-19 pandemic, in order to preserve the mental wellbeing of frontline providers and maximize safety, especially in case of pandemic resurgence $[7,18]$. Of note, the crisis apparently cemented most respondents' belief that their professional contribution could be relevant and did not alter the relationships with colleagues, indicating that radiology teams have faced the crisis in a cohesive manner.

A decrease of working hours before and during the crisis was reported, with private radiological facilities being perceived as more severely stricken and less protected by healthcare authorities compared to the public health system. While overall imaging volumes have shrunk (with a loss up to $80 \%$ of procedures at the height of the crisis), the private sector has been especially affected due to a reduced demand from patients, shunning medical services out of fear of contracting COVID-19 and over a period of economic setback [19-22]. This is in line with our finding of a higher proportion of respondents from private than from public facilities estimating a workload reduction greater than $50 \%$. Moreover, the fact that nonpublic radiology centers were commoner in hot regions could have resulted in greater damage to the healthcare system in areas more critically affected by the crisis.

A shift in workload has also been observed related to non-COVID-19 patients, with emergency procedures making up the most (about 70\%) of radiology workload. This change was associated to most radiologists' fear of having to catch up on deferred procedures and that the crisis could negatively impact the management of non-COVID-19 patients. Norbash et al. reported an overall volume drop of radiological procedures from 40 to $70 \%$ compared with the same period of the previous year, plummeting to $99 \%$ for breast screening examinations [19]. Boeken et al. reported a $61 \%$ volume drop in emergency CT examinations of non-COVID-19 patients performed some days before the COVID-19 lockdown compared to the same period of the previous year, and their finding of no difference in the distribution of positive CT scans would suggest that potentially severe conditions could go undiagnosed [22]. Still, in our survey the predominance of emergency and oncologic imaging in non-COVID-19 patients showed that radiology facilities have tried to guarantee life-critical procedures despite the crisis, in an attempt to minimize any potential major health hazard [23].

The overall impoverishment of radiological activity could have had a negative impact on the skills of training personnel, as perceived by most radiology residents. Concerns have been expressed by trainees and medical educators that the effectiveness of education programs could be jeopardized by social distancing, limited regular clinical and research activities with a reduced ability to perform live education sessions and hands-on practice, changes in residents' rotations or postponement of core examinations [24-26]. A perceived worse resident morale in radiology residency programs with redeployment was reported in a survey by the Well-Being Subcommittee of the Association of Program Directors in Radiology (APDR), along with a moderate/marked negative impact on the educational mission (70.1\% of respondents) and a mildly or markedly decreased morale of program directors (61\%) [24]. Some potential solutions have been proposed, including virtual meetings for e-learning and participation in remotely accessible research opportunities, simulated daily readout sessions during protected education time slots, reconfiguring rotations to ensure distancing while enabling preparation for delayed core 
exams, and avoiding multiple proceduralists for interventional procedures whenever feasible [24-29].

Attention should also be paid to the impact of the COVID-19 outbreak on the psychological status of radiology workers, resulting in a variable impairment that should be counteracted by taking appropriate actions [30-33]. Although psychological disturbances were most often tolerable as to not require specific treatment, moderate or even severe manifestations of depressive and/or anxiety symptoms occurred in a very consistent minority of respondents. Moreover, respondents from hot regions were more subject to some symptoms (including having negative thoughts or feeling restless or nervous), reflecting an association between exposure to the crisis and psychological damage. In a web-based survey conducted among Chinese healthcare workers during the COVID-19 pandemic, psychological issues were commoner in frontline workers, with a prevalence of anxiety, depression, insomnia and overall psychological problems of $46.04 \%, 44.37 \%, 28.75 \%$ and $56.59 \%$, respectively [31]. In a systematic meta-analysis including 13 studies and a combined total of 33,062 participants, the pooled prevalence of anxiety and depression was slightly higher than $20 \%$, whereas the same figure for insomnia was about $40 \%$ [32]. While females have been reported to have a higher risk of psychological symptoms [16, 32, 33], in our survey most symptoms had no gender prevalence, except for negative thoughts being commoner in women from hot regions. Interestingly, women from hot regions were less susceptible to feeling like living in slow motion than those from other regions, possibly suggesting a greater sense of control in workplaces more directly affected by the crisis, which could be a major driver of engagement and contribute to avoid burnout [34]. Moreover, in our survey the likelihood of having negative thoughts was higher among single-living respondents from hot regions compared to the same from other regions, supporting the role of social isolation as a potential risk factor for anxiety and depression in COVID-19 workers [33].

Our study has a potential limitation in that, although the participation rate was higher compared to previous SIRM surveys [11-14], only one fifth of SIRM members joined our survey, hence our findings might not be representative of the entire SIRM population. However, such circumstance seems unlikely because unfortunately the COVID-19 outbreak has been a nationwide emergency with similar problems across the country (albeit more severe in hot regions). It is also possible that our short survey duration of one week might have limited the number of participants and prevented us from obtaining further information. However, we restricted the survey duration to avoid the risk of bias due to collecting data over an extended time frame amid a rapidly changing emergency situation.

A further limitation is the lack of one or more reference groups of survey participants other than radiologists (including physicians from other specialties, technologists, nurses, and/or a general public outside the healthcare environment), potentially allowing to better discriminate findings specific to radiologists from those (e.g. symptoms of psychological distress) that might be shared by other groups. However, this would have involved splitting our survey (which contains many radiology-specific questions) into separate, more general parts to be administered to nonradiologists as well, thereby increasing its overall complexity and carrying the risk of bias due to inhomogeneity of data collected from different populations. While, in order to be meaningful, such an extended survey should have been performed simultaneously to that we actually conducted, we are planning on devising a further survey including a nonradiologist audience as a reference, in an attempt to corroborate our findings on a larger population from a wider perspective.

\section{Conclusions}

Our survey shows that the working and personal life of SIRM members has been impacted by the COVID-19 outbreak, with more specific patterns of involvement in hot regions due to their higher exposure. While our survey was restricted to radiologists and did not take into account other respondents' groups as a reference, we believe that specific recommendations should be drawn up based on our findings, including the following:

- To invest on professional training of all involved personnel, striving to provide universal access to PPE while fostering appropriate use of such equipment, thus maximizing safety and avoiding PPE misuse or waste.

- To encourage a responsible use of imaging in radiologists, referring clinicians and patients by improving the adherence to established guidelines and clinical decision support systems, in order to exploit available imaging resources in the most efficient manner while minimizing unnecessary procedures (and hence, potentially avoidable exposure of patients and staff to COVID-19 infection).

- To separate COVID-19 patients' pathways from those of other patients to the maximum possible extent, by dedicating specific environments and working shifts inside radiological departments and upgrading those latter to guarantee distancing, cleanliness and lack of contamination. 
- To increase the frequency of shift rotations for the entire radiological staff (including residents), to promote team working (as much as allowable in relation to distancing requirements and personnel availability) and to provide psychological support, in order to minimize the risk of burnout and psychological discomfort. Financial support should also be considered for private workers.

\section{Supplementary information}

The online version contains supplementary material available at https://doi. org/10.1186/s13244-021-00962-2.

Additional file 1. Survey form with answers in full for each question. *hot region.

\section{Abbreviations}

COVID-19: Coronavirus 2019 disease; PPE: Personal protective equipment.

\section{Authors' contributions}

FC, LF (equal contributors): study conceptualization, survey creation, data collection and analysis, interpretation of findings, preparation of manuscript draft. EN: software and statistical support, literature search, interpretation of findings, manuscript revision. RG: study conceptualization, interpretation of findings, manuscript revision, scientific guarantor of the study. VM: verification of methods and data integrity, interpretation of findings, manuscript revision. All authors read and approved the final manuscript.

\section{Funding}

The authors state that this work has not received any funding.

\section{Ethics approval and consent to participate}

Institutional Review Board approval was not required for this study because of its survey nature. The survey participants gave their implicit consent to the survey by accessing it and submitting their replies.

\section{Consent for publication}

Not applicable.

\section{Competing interests}

The authors declare that they have no competing interests.

\begin{abstract}
Author details
${ }^{1}$ Department of Radiology, IRCCS Azienda Ospedaliero-Universitaria di Bologna, Bologna, Italy. ${ }^{2}$ Department of Translational Research, University of Pisa, Via Roma, 67, 56126 Pisa, Italy. ${ }^{3}$ SIRM Foundation, Italian Society of Medical and Interventional Radiology, Milan, Italy. ${ }^{4}$ Department of Precision Medicine, University of Campania, Naples, Italy. ${ }^{5}$ Department of Radiology, Azienda Ospedaliero-Universitaria Careggi, Florence, Italy.
\end{abstract}

Received: 9 November 2020 Accepted: 4 January 2021 Published online: 17 February 2021

\section{References}

1. Guan W-J, Ni Z-Y, Hu Y et al (2020) Clinical characteristics of Coronavirus Disease 2019 in China. N Engl J Med 382:1708-1720

2. Hartley DM, Perencevich EN (2020) Public health interventions for COVID19: emerging evidence and implications for an evolving public health crisis. JAMA. https://doi.org/10.1001/jama.2020.5910

3. Barach P, Fisher SD, Adams MJ et al (2020) Disruption of healthcare: Will the COVID pandemic worsen non-COVID outcomes and disease outbreaks? Prog Pediatr Cardiol. https://doi.org/10.1016/j.ppedc ard.2020.101254

4. Mossa-Basha M, Meltzer CC, Kim DC, Tuite MJ, Kolli KP, Tan BS (2020) Radiology department preparedness for COVID-19: scientific expert review panel. Radiology 296:E106-E112

5. Rubin GD, Ryerson CJ, Haramati LB et al (2020) The role of chest imaging in patient management during the COVID-19 pandemic: a multinational consensus statement from the Fleischner Society. Radiology 296:172-180

6. Vagal A, Reeder SB, Sodickson DK, Goh V, Bhujwalla ZM, Krupinski EA (2020) The impact of the COVID-19 pandemic on the radiology research enterprise: scientific expert panel. Radiology. https://doi.org/10.1148/ radiol.2020201393

7. Siegal DS, Wessman B, Zadorozny J et al (2020) Operational radiology recovery in academic radiology departments after the COVID-19 pandemic: moving toward normalcy. J Am Coll Radiol. https://doi. org/10.1016/j.jacr.2020.07.004

8. ACR Coronavirus (COVID-19) resources. https://www.acr.org/Coron avirus-Covid-19-Resources. Accessed 21 Dec 2020

9. Important resources for radiologists regarding COVID-19. https://www. myesr.org/covid-19-resources. Accessed 21 Dec 2020

10. COVID-19 DATABASE. https://www.sirm.org/category/senza-categoria/ covid-19/. Accessed 21 Dec 2020

11. Coppola F, Bibbolino C, Grassi R et al (2016) Results of an Italian survey on teleradiology. Radiol Med 121:652-659

12. Faggioni L, Coppola F, Ferrari R, Neri E, Regge D (2017) Usage of structured reporting in radiological practice: results from an Italian online survey. Eur Radiol 27:1934-1943

13. Coppola F, Faggioni L, Grassi R et al (2019) Dematerialisation of patient's informed consent in radiology: insights on current status and radiologists' opinion from an Italian online survey. Radiol Med 124:846-853

14. Coppola F, Faggioni L, Regge D et al (2020) Artificial intelligence: radiologists' expectations and opinions gleaned from a nationwide online survey. Radiol Med. https://doi.org/10.1007/s11547-020-01205-y

15. ArcGIS Dashboards. http://opendatadpc.maps.arcgis.com/apps/opsda shboard/index.html\#/b0c68bce2cce478eaac82fe38d4138b1. Accessed 2 Sep 2020

16. Lai J, Ma S, Wang Y et al (2020) Factors associated with mental health outcomes among health care workers exposed to Coronavirus Disease 2019. JAMA Netw Open 3:e203976

17. Huang L, Wang Y, Liu J et al (2020) Factors associated with resilience among medical staff in radiology departments during the outbreak of 2019 Novel Coronavirus Disease (COVID-19): a cross-sectional study. Med Sci Monit 26:e925669

18. Mossa-Basha M, Linnau KF, Sahani DV (2020) Radiology department preparedness in the Coronavirus Disease 2019 (COVID-19) postshutdown environment. J Am Coll Radiol 17:890-893

19. Norbash AM, Moore AV Jr, Recht MP et al (2020) Early-stage radiology volume effects and considerations with the Coronavirus Disease 2019 (COVID-19) pandemic: adaptations, risks, and lessons learned. J Am Coll Radiol. https://doi.org/10.1016/j.jacr.2020.07.001

20. Sharpe RE Jr, Kuszyk BS, Mossa-Basha M, RSNA COVID-19 Task Force, (2020) Special Report of the RSNA COVID-19 Task Force: the short- and long-term financial impact of the COVID-19 pandemic on private radiology practices. Radiology. https://doi.org/10.1148/radiol.2020202517

21. Cavallo JJ, Forman HP (2020) The economic impact of the COVID-19 pandemic on radiology practices. Radiology. https://doi.org/10.1148/ radiol.2020201495

22. Boeken T, Le Berre A, Mebazaa A, Boulay-Coletta I, Hodel J, Zins M (2020) Non-COVID-19 emergencies: where have all the patients gone? Eur Radiol. https://doi.org/10.1007/s00330-020-06961-z

23. Rosenbaum $L$ (2020) The untold toll - the pandemic's effects on patients without Covid-19. N Engl J Med 382:2368-2371

24. Alvin MD, George E, Deng F, Warhadpande S, Lee SI (2020) The impact of COVID-19 on radiology trainees. Radiology 296:246-248

25. Roytman M, Shah S (2020) Lessons learned during the COVID-19 pandemic: a single institution radiology chief resident experience. Clin Imaging 68:90-93

26. Warhadpande S, Khaja MS, Sabri SS (2020) The impact of COVID-19 on interventional radiology training programs: what you need to know. Acad Radiol 27:868-871 
27. Robbins JB, England E, Patel MD et al (2020) COVID-19 Impact on wellbeing and education in radiology residencies: a survey of the Association of Program Directors in Radiology. Acad Radiol 27:1 162-1172

28. Matalon SA, Souza DAT, Gaviola GC, Silverman SG, Mayo-Smith WW, Lee LK (2020) Trainee and attending perspectives on remote radiology readouts in the era of the COVID-19 pandemic. Acad Radiol 27:1147-1153

29. Recht MP, Fefferman NR, Bittman ME et al (2020) Preserving radiology resident education during the COVID-19 pandemic: the simulated daily readout. Acad Radiol 27:1154-1161

30. Chew NWS, Lee GKH, Tan BYQ et al (2020) A multinational, multicentre study on the psychological outcomes and associated physical symptoms amongst healthcare workers during COVID-19 outbreak. Brain Behav Immun 88:559-565

31. Que J, Shi L, Deng J et al (2020) Psychological impact of the COVID-19 pandemic on healthcare workers: a cross-sectional study in China. Gen Psychiatr 33:e100259
32. Pappa S, Ntella V, Giannakas T, Giannakoulis VG, Papoutsi E, Katsaounou P (2020) Prevalence of depression, anxiety, and insomnia among healthcare workers during the COVID-19 pandemic: a systematic review and metaanalysis. Brain Behav Immun 88:901-907

33. Luo M, Guo L, Yu M, Jiang W, Wang H (2020) The psychological and mental impact of coronavirus disease 2019 (COVID-19) on medical staff and general public - a systematic review and meta-analysis. Psychiatry Res 291:113190

34. Wu Y, Wang J, Luo C et al (2020) A comparison of burnout frequency among oncology physicians and nurses working on the frontline and usual wards during the COVID-19 epidemic in Wuhan, China. J Pain Symptom Manage 60:e60-e65

\section{Publisher's Note}

Springer Nature remains neutral with regard to jurisdictional claims in published maps and institutional affiliations.

\section{Submit your manuscript to a SpringerOpen ${ }^{\circ}$ journal and benefit from:}

- Convenient online submission

- Rigorous peer review

- Open access: articles freely available online

- High visibility within the field

- Retaining the copyright to your article

Submit your next manuscript at $\boldsymbol{\nabla}$ springeropen.com 